\title{
Laboratory plasma physics experiments using merging supersonic plasma jets
}

\author{
S. C. $\mathrm{Hsu}^{1} \dagger$, A. L. Moser ${ }^{1} \ddagger$, E. C. Merritt ${ }^{1,2}$, C. S. Adams ${ }^{1,2}$, J. P. Dunn ${ }^{1}$, \\ S. Brockington ${ }^{3}$, A. Case ${ }^{3}$, M. Gilmore ${ }^{2}$, A. G. Lynn ${ }^{2}$, S. J. Messer ${ }^{3}$ q \\ and F. D. Witherspoon ${ }^{3}$ \\ ${ }^{1}$ Los Alamos National Laboratory, Los Alamos, NM 87545, USA \\ ${ }^{2}$ University of New Mexico, Albuquerque, NM 87131, USA \\ ${ }^{3}$ HyperV Technologies Corp., Chantilly, VA 20151, USA
}

(Received 1 August 2014; revised 21 October 2014; accepted 7 November 2014; first published online 10 December 2014)

We describe a laboratory plasma physics experiment at Los Alamos National Laboratory that uses two merging supersonic plasma jets formed and launched by pulsed-power-driven railguns. The jets can be formed using any atomic species or mixture available in a compressed-gas bottle and have the following nominal initial parameters at the railgun nozzle exit: $n_{e} \approx n_{i} \sim 10^{16} \mathrm{~cm}^{-3}, T_{e} \approx T_{i} \approx 1.4 \mathrm{eV}$, $V_{\text {jet }} \approx 30-100 \mathrm{~km} / \mathrm{s}$, mean charge $\bar{Z} \approx 1$, sonic Mach number $M_{s} \equiv V_{\text {jet }} / C_{s}>10$, jet diameter $=5 \mathrm{~cm}$, and jet length $\approx 20 \mathrm{~cm}$. Experiments to date have focused on the study of merging-jet dynamics and the shocks that form as a result of the interaction, in both collisional and collisionless regimes with respect to the inter-jet classical ion mean free path, and with and without an applied magnetic field. However, many other studies are also possible, as discussed in this paper.

\section{Introduction}

The Plasma Liner Experiment (PLX) is a laboratory plasma physics facility at Los Alamos National Laboratory. The primary purpose and goal of the PLX is to form and study spherically imploding plasma liners via multiple merging plasma jets, motivated by high-energy-density (HED) physics and magneto-inertial fusion (MIF) applications (Thio et al. 1999, 2001; Hsu et al. 2012a,b; Santarius 2012; Knapp and Kirkpatrick 2014). For fundamental HED-physics studies (Drake 2006), PLX is envisioned to provide an economic means for forming inertially confined $\mathrm{cm}-, \mu \mathrm{s}-$, and Mbar-scale plasmas (Awe et al. 2011; Davis et al. 2012; Cassibry et al. 2013) upon the stagnation of up to 60 merged pulsed-power-driven, supersonic plasma jets. Such an experiment would allow for good diagnostic measurements on largerspatial and longer-temporal scales and a much higher shot rate than those of typical laser- or Z-pinch-driven HED experiments. For the MIF application (Lindemuth and Kirkpatrick 1983; Kirkpatrick et al. 1995; Lindemuth and Siemon 2009), the use of a spherically converging plasma liner for compressing a magnetized target plasma would avoid the repetitive hardware destruction inherent in solid-liner approaches (e.g. Intrator et al. 2004; Slutz et al. 2010; Degnan et al. 2013), and it would allow

$\dagger$ Email address for correspondence: scotthsu@lanl.gov

$\mp$ Now at General Atomics, San Diego, CA.

I Now at Foreground Security, Herndon, VA. 
for a higher repetition rate $(\sim 1 \mathrm{~Hz})$, which is desirable for an economic, pulsed fusion-energy system.

The PLX facility was built in 2010-2011, and the first plasma jet was fired in September, 2011. Initial research characterized in detail the plasma properties and evolution of a single jet (Hsu et al. 2012b). A second railgun was installed in mid-2012, and ensuing experiments focused on the oblique merging of two plasma jets, resulting in detailed observations shown to be consistent with collisional plasma shocks (Merritt et al. 2013, 2014). Due to loss of funding, we were not able to build the facility up to 30 railguns as originally planned. Instead, ongoing experiments are focused on the head-on merging of two plasma jets to form and study unmagnetized and magnetized plasma shocks. An in-chamber pulsed Helmholtz $(\mathrm{HH})$ coil was installed in late 2013 to enable the study of magnetized shocks. The facility now enables a unique experimental research program on the detailed study of plasma shocks, from collisional-to-collisionless and unmagnetized-to-magnetized regimes. Preliminary designs for a 36 - or 60 -jet experiment (with $\sim 1.5 \mathrm{MJ}$ of capacitive stored energy) to form spherically imploding plasma liners are also in hand.

Plasma jet and shock studies on PLX complement many other related, contemporary experimental efforts. Examples include studies of plasma jet dynamics, interactions, and/or shock formation in astrophysically or fusion-relevant contexts using a variety of plasma formation methods, ranging from coaxial plasma guns (e.g. Bellan et al. 2005; Hsu and Bellan 2005; Moser and Bellan 2012) to wire-array-driven pinches (e.g. Haas et al. 2011; Gourdain and Seyler 2013; Swadling et al. 2014) to laser-driven experiments (e.g. Fox et al. 2013; Li et al. 2013; Ross et al. 2013; Fiksel et al. 2014). In general, PLX plasmas are colder and more collisional than the other experiments, but have the benefits of larger spatial size for ease of diagnostic measurements, more choices for the working plasma species, and the option of both unmagnetized and magnetized experiments. A brief overview of many plasma-jet experiments and formation methods is given in Hsu (2009).

The rest of this paper is organized as follows. Section 2 describes the PLX experimental device and parameters. Section 3 discusses some of the physics questions that can be addressed on PLX. Section 4 summarizes the major results obtained thus far, and Sec. 5 describes some future opportunities.

\section{Description of experimental device and parameters}

\subsection{Device description}

The PLX consists of a $9 \mathrm{ft}$. (2.74 m) diameter spherical vacuum chamber (Fig. 1), two pulsed-power-driven plasma railguns (Sec. 2.1.1), a diagnostic suite (Sec. 2.1.2), and an in-chamber $\mathrm{HH}$ coil (Sec. 2.1.4) to generate a pulsed magnetic field up to $0.22 \mathrm{~T}$. The facility is housed in a $3000 \mathrm{ft}^{2}\left(279 \mathrm{~m}^{2}\right)$ high-bay space with a 10-ton $(9072 \mathrm{~kg})$ bridge crane, roll-up door with 18.5-ft. $(5.6 \mathrm{~m})$ tall and $16-\mathrm{ft}$. $(4.9 \mathrm{~m})$ wide clearance, 208- and 480-VAC power, and building chilled water and compressed air.

\subsubsection{Plasma railguns and pulsed-power systems.}

The plasma railguns (Figs 2(a) and (b)) and their high-current, spark-gap switches (Fig. 2(c)) were designed and built by collaborator HyperV Technologies Corp. The railgun has two parallel-plate electrodes made of tungsten alloy (HD-17BB), separated by insulators made of zirconium-toughened alumina (ZTA). The railgun bore is 1 in. $(2.54 \mathrm{~cm}) \times 1$ in. $(2.54 \mathrm{~cm})$, and the body is a clamshell design consisting of two Noryl halves bolted together (Figs 2(a) and (b)). A custom-built gas-puff valve injects 


\begin{tabular}{lcccc}
\hline & $\begin{array}{c}\text { Capacitor } \\
\text { model }\end{array}$ & $\begin{array}{c}\text { Rated bank } \\
\text { voltage }(\mathrm{kV})\end{array}$ & $\begin{array}{c}\text { Total bank } \\
\text { capacitance }(\mu \mathrm{F})\end{array}$ & $\begin{array}{c}\text { Max. } \\
\text { energy (kJ) }\end{array}$ \\
Railguns (one bank per gun) & Maxwell 32 184 & 40 & 12 or 36 & 9.6 or 28.8 \\
Pre-ionization (for both guns) & Maxwell 32814 & 40 & 12 & 9.6 \\
Gas valve (for both guns) & Maxwell 32 567 & 20 & 24 & 1.7 \\
HH coil & GA 32934 & 5 & 4000 & 50
\end{tabular}

TABLE 1. Capacitor bank parameters.

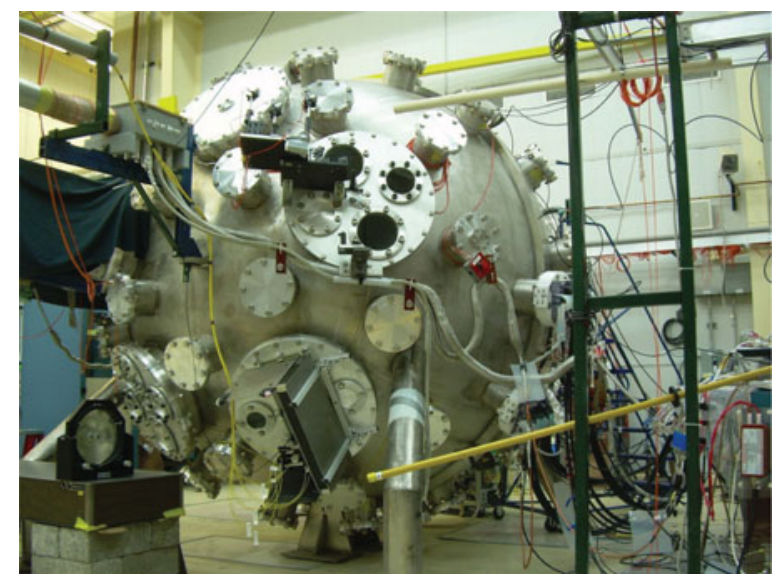

FIGURE 1. Photograph of the PLX vacuum chamber (2.74-m diameter).

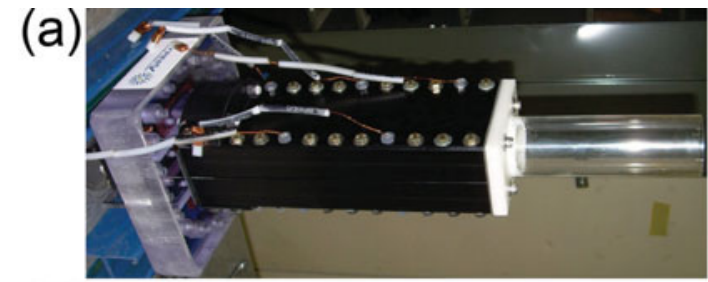

(b)
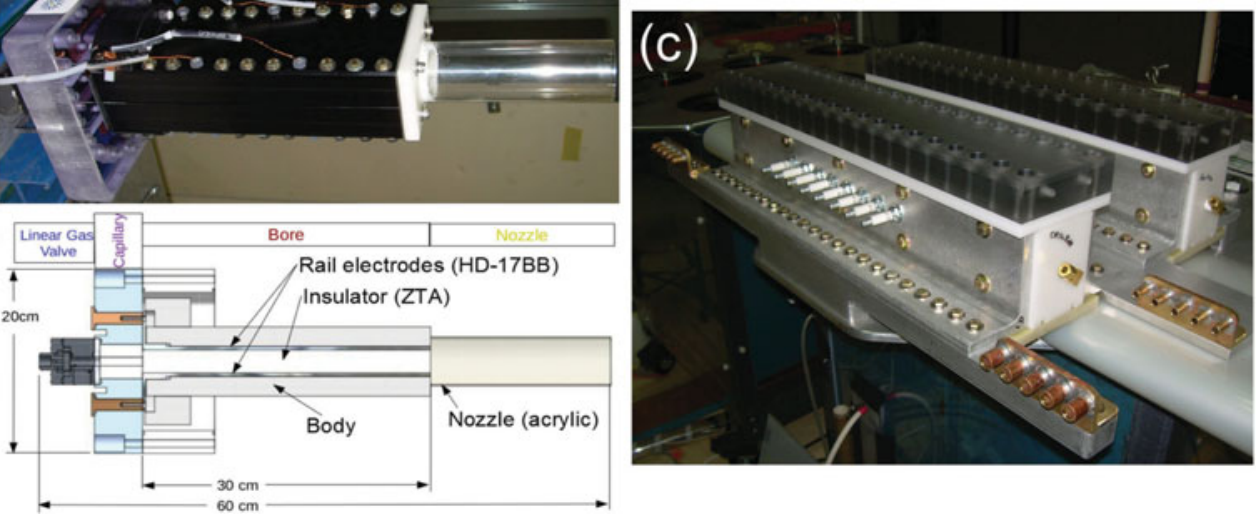

FiguRE 2. (a) Photograph of railgun. (b) Schematic of railgun (courtesy of HyperV Technologies). (c) Photograph of two linear, spark-gap switches (one main switch, one crowbar) installed on top of a railgun capacitor bank.

neutral gas into a pre-ionization (PI) chamber at the rear of the railgun. A cylindrical acrylic nozzle with $5 \mathrm{~cm}$ inner diameter is mounted at the exit of the railgun bore. The rails, PI, and gas valves $(\mathrm{GV})$ are driven by four separate capacitor banks, which are summarized in Table 1. The GVs and PIs of both guns are connected in parallel and driven by a single GV and PI bank, respectively. 


\subsubsection{Diagnostics.}

Operational diagnostics include a multi-chord visible interferometer, a visible-tonear-infrared survey spectrometer, an array of three photodiodes (PD), a chargecoupled-device (CCD) camera, a schlieren imaging system, an electrostatic triple probe, and magnetic probe arrays. We briefly summarize the diagnostic capabilities here; more technical details are given elsewhere (Lynn et al. 2010; Hsu et al. 2012b).

The 561-nm multi-chord interferometer (Merritt et al. 2012a,b) is fiber-coupled between the launch laser optics and the vacuum chamber, allowing for relatively easy (a few days work) modification of chord positioning for different experiments and different plasma gun geometries. Several different chord arrangements have been used for single-jet propagation (Hsu et al. 2012b), two-jet oblique-merging experiments (Merritt et al. 2013, 2014), and two-jet head-on merging experiments (Moser and Hsu 2014). The time-resolution of the continuous interferometer phase signals is $50 \mathrm{~ns}$, limited by the $40-\mathrm{MHz}$ digitization rate. Spatial resolution is determined by the chord spacing (typically a few $\mathrm{cm}$ ) and the laser spot size $(\approx 3 \mathrm{~mm}$ diameter at the position of the plasma).

The survey spectrometer system consists of a $0.275-\mathrm{m}$ spectrometer and a gated (0.45 $\mu$ s duration), 1024-pixel, multi-channel-plate array covering the wavelength range $300-900 \mathrm{~nm}$. The best spectral resolution, corresponding to a $600-1$ ine $/ \mathrm{mm}$ grating, is $0.152 \mathrm{~nm} /$ pixel. Plasma light is collected at a vacuum chamber window into a 5-mm diameter collimating lens and brought to the spectrometer with an optical fiber bundle. The diameter of the spectrometer viewing chord is $\approx 7 \mathrm{~cm}$ at the position of the plasma. The lens and fiber can be moved easily to different viewing positions.

A three-channel PD array collects chord-integrated and time-resolved broadband visible $(300-850 \mathrm{~nm})$ plasma emission for determining jet propagation speed, based on the viewing-chord positions and time-of-flight estimates of features in the PD data traces. The peak PD responsivity is $0.65 \mathrm{~A} / \mathrm{W}$ at $970 \mathrm{~nm}$, and the frequency response decreases with increasing gain $(2.1$ and $0.1 \mathrm{MHz}$ at 20 and $50 \mathrm{~dB}$, respectively). The light is collected through an adjustable aperture that is typically set at $1 \mathrm{~cm}$, which constitutes the nominal spatial resolution.

An intensified CCD camera (DiCam Pro ICCD), with spectral sensitivity from the UV to near-IR, is used to capture time-gated, visible images of the experiment. The camera records $1280 \times 1024$ pixel images with 12-bit dynamic range. The typical gate time used is $20 \mathrm{~ns}$. The camera is triggered remotely via an optical fiber, housed inside a metal shielding box, and mounted next to a large rectangular borosilicate window on the vacuum chamber (although it can be moved to other ports as well).

A schlieren system (Settles 2001) for obtaining 2D images $(4 \mathrm{~cm} \times 16 \mathrm{~cm})$ sensitive to plasma-density gradients has been constructed. The PLX schlieren system (Adams et al. 2012) uses a pulsed 1.064- $\mu \mathrm{m} \mathrm{Nd:YAG} \mathrm{laser} \mathrm{(3} \mathrm{ns} \mathrm{pulse,} \mathrm{30-80} \mathrm{mJ)} \mathrm{expanded}$ to a $20-\mathrm{cm}$ diameter beam that is then collimated and passed through the plasma between $25-\mathrm{cm}$ diameter, $f / 8$ mirrors. A tunable knife edge blocks light refracted by density gradients in the plasma. The image is captured by an IR camera (Apogee Alta U1109) which contains a $2048 \times 512 \mathrm{CCD}$ detector with $8 \%$ quantum efficiency at $1.064 \mu \mathrm{m}$. The system is designed to give images with $>5 \%$ contrast for electrondensity gradients $\gtrsim 10^{15} \mathrm{~cm}^{-4}$, i.e. for 1 -cm-scale gradients in the presence of $10^{14}$ $\mathrm{cm}^{-3}$ density plasmas. The intensity of the recorded image is proportional to $\nabla N$, where $N$ is the refractive index of the plasma. The PLX schlieren diagnostic has yet to be tested on plasmas with sufficient density gradients to yield images with detectable contrast. 
Magnetic probe arrays measure all three components of the magnetic field along the railgun nozzle and within the plasma-jet-interaction region. The nozzle probe array consists of hand-wound coils (using $0.5 \mathrm{~mm}$ diameter Kapton-coated magnet wire) around a rectangular Delrin rod $\left(0.37 \times 0.48 \mathrm{~cm}^{2}\right)$. These probes have effective turns $\times$ area $(N A)$ of $\approx 10 \mathrm{~cm}^{2}$ in the frequency range $10-5000 \mathrm{kHz}$. The internal probe array is mounted inside an insertable probe shaft and contains two sets of 3-axis coils at two positions separated by $27.75 \mathrm{~cm}$, for the simultaneous measurement of up to six $B$-dot signals simultaneously. These probe arrays use commercial inductor chips (Coilcraft $1008 \mathrm{CS}-272 \mathrm{XJLB}$, with dimensions $2.92 \times 2.79 \times 2.03 \mathrm{~mm}^{3}$ ) ; the probe construction is similar to that described by Romero-Talamás et al. (2004). The internal probe array coils have $N A \approx 0.7 \mathrm{~cm}^{2}$ between $10-1000 \mathrm{kHz}$. The $B$-dot signals are integrated by a passive integration circuit with $R C=100 \mu \mathrm{s}$.

An electrostatic triple probe (e.g. Ji et al. 1991) measures the instantaneous floating potential $V_{f}$, electron temperature $T_{e}$, and ion saturation current $I_{\text {sat }}$ as a function of time at one position in space. The probe can be moved to different radial positions with respect to chamber center. Due to the lack of a reliable, nearby reference ground potential (due to large voltage excursions on the vacuum chamber during a shot), the triple probe is not yet producing reliable data. To improve the probe operation, we have built four battery-powered, analog optical channels (up to 1-MHz frequency response) to bring probe signals back to the data acquisition system.

Finally, there are Rogowski coils, high-voltage probes, voltage divider networks, and pulsed current monitors to record the pulsed electrical currents and voltages of all the capacitor banks.

\subsubsection{Control and data acquisition systems.}

The PLX has an FPGA-based control/trigger system and CAMAC-based digitaldata-acquisition system, all integrated through a LabVIEW software interface and operated from a desktop computer. Both analog and optical channels are available for diagnostic triggering and operating relays for the various hardware sub-systems. The data-acquisition system consists of 64 channels with 12-bit resolution, up to $40 \mathrm{MHz}$ sampling rate, and 32 kilosamples of memory per channel (four Joerger TR modules). Shot information and diagnostic data are stored in an MDSplus tree (www.mdsplus.org).

\subsubsection{In-chamber $H H$ coils.}

We have installed inside the vacuum chamber a pair of magnetic coils arranged in a $\mathrm{HH}$ configuration (coil radius and separation $\approx 30 \mathrm{~cm}$ ) inside the vacuum chamber (see Fig. 3). The coils and mounting structure were designed and fabricated by Woodruff Scientific, LLC, and are presently installed such that the $\mathrm{HH}$ field is perpendicular to the direction of jet propagation, but the $\mathrm{HH}$ field can be adjusted up to $\pm 30^{\circ}$ about the perpendicular and parallel directions with respect to the jet propagation direction. The coils are driven by a capacitor bank (see Table 1) with a current-rise time of $\approx 1.3 \mathrm{~ms}$. The maximum $\mathrm{HH}$-bank operating voltage of $4 \mathrm{kV}$ gives a coil current $\approx 8.2 \mathrm{kA}$ and a peak magnetic field of $0.22 \mathrm{~T}$. The minimum operating voltage is around $200 \mathrm{~V}$ (corresponding to about $0.44 \mathrm{kA}$ and $0.012 \mathrm{~T}$ peak magnetic field), below which the $\mathrm{HH}$-bank ignitron switch does not fire reliably. The magnetic-field profiles have been characterized experimentally using three concentric flux loops of different radii. 


Parameter
Ion+neutral density $n_{\text {tot }}\left(\mathrm{cm}^{-3}\right)$
Electron temperature $T_{e}(\mathrm{eV})$
Jet velocity $V(\mathrm{~km} / \mathrm{s})$
Mean charge $\bar{Z}$
Jet length $L(\mathrm{~cm})$
Jet diameter $D(\mathrm{~cm})$

$Z \approx 2 \mathrm{~cm}$
$2 \times 10^{16}$
1.4
30
0.96
20
5

5

$\begin{array}{cc}Z \approx 41 \mathrm{~cm} & \text { range } \\ 2 \times 10^{15} & 10^{14}-10^{17} \\ 1.4 & 1-3 \\ 30 & 30-100 \\ 0.94 & \sim 1 \\ 45 & \\ 10-20 & \end{array}$

TABLE 2. Measured and achievable range of railgun-driven argon plasma jet parameters. Hydrogen and helium jets have similar values.

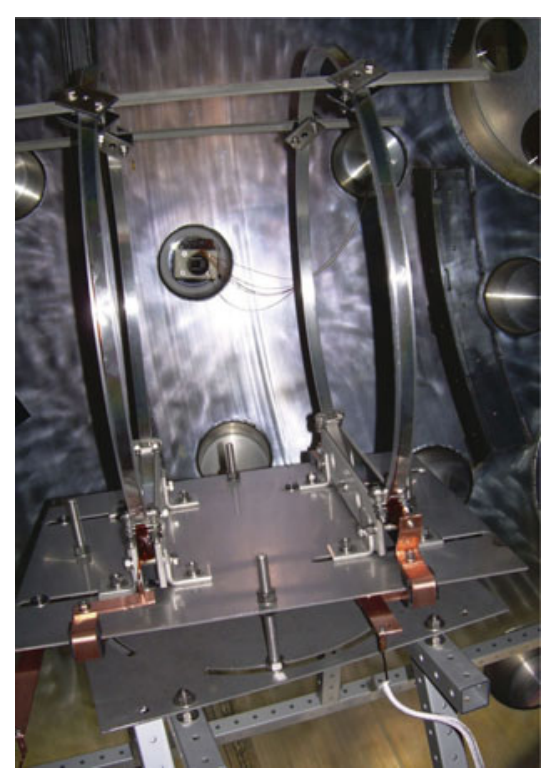

FIGURE 3. Photograph of the in-chamber $\mathrm{HH}$ coil, looking down the barrel of one railgun.

\subsection{Parameters}

Table 2 summarizes the experimentally measured or inferred physical parameters of our plasma jets, both at the gun-nozzle exit and after propagating $\approx 41 \mathrm{~cm}$. For head-on jet-merging experiments, the jets propagate $\approx 1.1 \mathrm{~m}$ before colliding with each other near chamber center, where the jet $n_{\text {tot }} \sim 10^{14} \mathrm{~cm}^{-3}$ and $T_{e} \approx 1 \mathrm{eV}$ just before the collision. For the achievable range of $n_{t o t}, T_{e}$, and $V$ just before jets collide, the inter-jet counter-streaming ion mean free path $\lambda_{\mathrm{i}, \mathrm{ctr}}$ (mean free path of an ion in one jet interacting with all species in the opposing jet) can range from collisional, i.e. $\lambda_{\mathrm{i} \text {,ctr }}<\Delta$ (Merritt et al. 2013, 2014), where $\Delta$ is the gradient-scale-length of the shock that forms as a result of the colliding jets, to collisionless, i.e. $\lambda_{\mathrm{i}, \mathrm{ctr}} \gg \Delta$ (the goal of ongoing work). Note that both ion and electron collisionality are very high within each jet, and that the inter-jet counter-streaming electron mean free path is very short, i.e. $\lambda_{\text {e,ctr }} \ll \Delta$. 


\section{Physics questions that can be addressed}

\subsection{Plasma shock structure and dynamics}

Highly supersonic plasmas, i.e. in which the sonic Mach number $M \equiv V / C_{s} \gg 1$ (with $V$ the plasma flow speed and $C_{s}$ the ion sound speed), are needed and provide a natural opportunity to study plasma shock physics (e.g. Jaffrin and Probstein 1964; Casanova et al. 1991; Sagdeev and Kennel 1991). Detailed laboratory studies of the latter have been scarce for two main, coupled reasons: (i) the most readily available ways to form supersonic plasmas tended to be in very high-density regimes, e.g. using laser, $z$-pinch, or $\theta$-pinch sources that (ii) presented significant diagnostic challenges. The latter arose due to a combination of small spatial scales, high plasma densities, and/or lack of diagnostic access. The PLX, with its macroscopic plasma jets at moderate density and completely open diagnostic access, presents a unique experimental platform for studying detailed plasma shock structure and dynamics on multiple-cm spatial and several- $\mu$ s time scales. The experiment also offers the ability to span collisional to collisionless regimes for the counter-streaming ions and unmagnetized to magnetized jet interactions.

\subsection{Equation of state in regimes intermediate between magnetic and inertial fusion}

Plasma equation of state (EOS), i.e. ionization state and the relationship between specific internal energies and pressure, can be complicated depending on the regime of plasma temperature and density as well as the plasma species or mixture of species. In magnetic fusion plasmas, which typically are at low density and consist of fully ionized hydrogen isotopes, EOS is relatively straightforward, e.g. governed by the ideal gas law $p=n_{e} k T_{e}+n_{i} k T_{i}$ with $n_{e}=n_{i}$. On the other hand, in inertial fusion and HED plasmas, which can have mixed-species plasmas with less than fully stripped ions, EOS is a leading order issue complicated by atomic physics, and significant effort has been made and sophisticated capabilities developed to model EOS.

The PLX railguns can generate low-to-moderate density plasmas using any species or mixture available in a compressed-gas bottle. The density and temperature range (see Table 2) are such that the mean charge state $\bar{Z}$ is rapidly varying, and localthermodynamic-equilibrium (LTE) and non-LTE models predict notably different EOS. PLX plasmas, whether single- or merging-jet, offer a platform for measuring and constraining EOS in a plasma regime that has not received much detailed attention.

\subsection{Interaction of plasma flow with vacuum magnetic field or magnetized plasma}

Studies of plasma flow across a magnetic field (e.g. Baker and Hammel 1965), or into another magnetized plasma (e.g. Liu and Hsu 2011) have a long history, and have been motivated by both basic plasma physics and applications such as tokamak fueling (e.g. Perkins et al. 1988). With our in-chamber HH coil, we can study supersonic plasma jet interactions with a vacuum magnetic field. In addition, because our plasma jets each consist of multiple 'blobs' due to the ringing railgun current, we can study the interaction of trailing blobs with stagnated magnetized plasma formed from the merged leading blobs.

\section{Summary of major results}

\subsection{Characterization of single-jet parameters and evolution}

The first set of experiments that we performed on PLX focused on the detailed characterization of the plasma parameters, profiles, and evolution over $\approx 1 \mathrm{~m}$ of 
propagation of a single argon plasma jet launched by a plasma railgun (Hsu et al. 2012b). These experiments were done to help us understand and interpret planned, subsequent experiments based on the merging of two or more plasma jets, and also to provide accurate initial conditions for numerical modeling of experiments using these plasma jets. These experiments also contributed uniquely to the plasma railgun literature, most of which has focused on in-bore characterization of the plasma armature as a pusher for launching solid projectiles to high velocities (see Batteh 1991, for example). Table 2 summarizes the measured (or experimentally inferred) argon plasma jet parameters at the exit of the railgun nozzle $(Z \approx 2 \mathrm{~cm})$ and downstream $(Z \approx 41 \mathrm{~cm})$, as well as the expected range of values for some of the parameters, although the latter were not systematically measured in the single-jet studies.

The plasma jet is essentially unmagnetized after a few tens of centimeters of propagation (at a typical speed of 3-7 cm/ $/ \mu \mathrm{s}$ ) due to fast resistive decay of the magnetic field that is embedded in the jet as it exits the railgun bore. Using magnetic pickup coils mounted on the exterior surface of the railgun nozzle, we have measured (Merritt et al. 2014) the decay time of the transverse (relative to the jet propagation) component of the magnetic field (of order $750 \mathrm{G}$ near the railgun nozzle exit for peak railgun current of order $200 \mathrm{kA}$ ) to be $\approx 5.6 \mu \mathrm{s}$, which is consistent with the expected magnetic diffusion time estimated using the plasma jet parameters and radial scale lengths. Thus, for the jet-merging studies described in Secs 4.2 and 4.3, the jet kinetic energy density $\left(\rho v^{2} / 2\right)$ is much greater (by a factor of $\left.50-10^{4}\right)$ than the magnetic energy density $\left(B^{2} / 2 \mu_{0}\right)$ at the time of initial jet merging.

\subsection{Evidence for collisional shock formation between obliquely merging plasma jets}

Following the single-jet experiments, we conducted experiments on the oblique merging of two plasma jets (Merritt et al. 2013, 2014), in order to gain better understanding of the essential underlying physical process for plasma liner formation via merging plasma jets. This work led to the identification and characterization of the stagnation layer between obliquely merging jets and the demonstration that the stagnation layer morphology is consistent with that of a collisional oblique plasma shock (Merritt et al. 2013, 2014).

We obtained temporally and spatially resolved survey-spectrometer and interferometer measurements of the stagnation layer, along with sequences of time-gated visible CCD images. Based on experimentally inferred plasma parameters, the interjet ion mean free path were less than the stagnation layer thickness, i.e. the oblique jet-merging was in a collisional regime. Detailed analyses of the data from all three diagnostics allowed us to arrive at the following key results (Merritt et al. 2013, 2014): demonstration that (a) the observed stagnation layer morphology is consistent with that expected of a collisional oblique plasma shock, (b) the peak density exceeds that of simple plasma interpenetration but is about a factor of 2 smaller than the peak density predicted by hydrodynamic shock theory, and (c) the observed gradient scale length at the postulated shock boundary is consistent with two-fluid plasma theory predictions of shock layer thickness.

\subsection{Transition from collisionless interpenetration to collisional stagnation during head-on jet merging}

In working toward a more collisionless regime for the counter-streaming ions between the merging jets, we performed a set of head-on jet-merging experiments in which the interaction between jets began with collisionless interpenetration but then transitioned to collisional stagnation due to a rising $\bar{Z}$ (Moser and Hsu 2014). This observation demonstrates a clear way by which an initially collisionless interaction between 
colliding plasmas can evolve toward a collisional one, and provides data that can be used to validate physics models of plasma collisionality and ionization in the presence of complex EOS. The observation of unmagnetized, supersonic interpenetration also establishes a regime in which neither macro- nor micro-instabilities are present or strong enough to introduce collisionless shock effects.

\subsection{Observation of Rayleigh-Taylor-instability growth and progression to longer wavelength}

As mentioned earlier, our ringing railgun current produces several serial plasma 'blobs', each separated in time by 20-30 $\mu$ s. The leading blob of each jet each compresses the applied magnetic field and stagnates in the center of the chamber upon merging with the opposing jet. We observed one trailing blob decelerating against the stagnated magnetized plasma in the center (formed by the merged leading blobs). Images from a multiple-frame CCD camera (Invisible Vision model UHSi 12/24) revealed the growth of Rayleigh-Taylor-like fingers at the leading edge of this trailing blob as well as the evolution toward longer wavelength of these fingers. Preliminary instability-growth-rate estimates and magnetohydrodynamic (MHD) simulations suggest that our observations are consistent with RayleighTaylor instability of this decelerating interface. The progression toward longer mode wavelength could be due to a combination of magnetic stabilization and viscous damping. Detailed analysis is ongoing, and these results will be reported elsewhere.

\subsection{Ongoing studies of magnetized head-on jet merging to study cosmically relevant} collisionless shocks

Ongoing experiments on PLX are aimed at forming and studying 'cosmically relevant' collisionless shocks, as described by Drake (2000), who argues that such experiments must meet a number of competing constraints on magnetization, plasma $\beta$, Alfvén Mach number $M_{A}$, and collisionality. Table 3 summarizes the original design parameters for collisionless shock studies on PLX using head-on-merging plasma jets. Hybrid particle-in-cell (PIC) and fully kinetic PIC simulations of head-on merging jets in this parameter regime confirmed that we would be in a collisionless regime and that magnetized shocks should form (Thoma et al. 2013). However, the level of impurities in our railgun-driven jets was higher than expected (Hsu et al. 2012b; Merritt et al. 2014; Moser and Hsu 2014), leading to an unanticipated rise in $\bar{Z}$ (even in hydrogen experiments), causing a reduction in the counter-streaming ion-ion mean free path that scales as $\bar{Z}^{-4}$, and a transition to a collisional regime (described in Sec. 4.3) at the originally chosen jet velocities. Thus, ongoing work is aimed at reaching collisionless regimes for the counter-streaming ions by achieving even higher counter-streaming jet velocities to overcome the rising- $\bar{Z}$ effect. Failing this, we may need to upgrade to coaxial guns (Witherspoon et al. 2009), which are expected to have far lower levels of impurities.

\section{Future opportunities}

Future opportunities on PLX with two plasma guns include further and more detailed studies of all the topics discussed in Secs 3 and 4. Upgraded coaxial guns (e.g. Witherspoon et al. 2009) would reduce impurities and allow for higher velocities than railguns. In addition, the plasma shock and interpenetration data offer a rare opportunity to validate collisionality, EOS, ionization, and shock-handling physics models employed in MHD, multi-fluid, and kinetic plasma codes. 


Parameter
Species
Density $\left(\mathrm{cm}^{-3}\right)$
Temperature $(\mathrm{eV})$
Speed $(\mathrm{km} / \mathrm{s})$
Magnetic field $(\mathrm{G})$
Length $L(\mathrm{~cm})$
Radius $R(\mathrm{~cm})$
Criterion
$2 R / \rho_{i} \gg 1$
$\beta>1$
$M_{A}>1$
$\lambda_{i} / \rho_{i} \gg 1$
$R_{M} \gg 1$
$\omega_{c i} \tau_{\exp } \gg 1$
$L /\left(c / \omega_{p i}\right) \gg 1$

$$
\begin{gathered}
\text { Jets at collision } \\
\mathrm{H}^{+} \\
3 \times 10^{14} \\
1 \\
V_{\mathrm{jet}}=100 \\
300 \text { (applied) } \\
50 \\
15
\end{gathered}
$$

Estimate for proposed experiment

(post-shock values)

$$
\begin{gathered}
30 \\
4.7 \\
2.2 \\
28 \\
\sim 100\left(\text { for } T_{e} \sim 10 \mathrm{eV}\right) \\
34 \\
76
\end{gathered}
$$

TABLE 3. Proposed reference experimental values, and evaluation of relevant physics criteria against those of Drake (2000) for a cosmically relevant collisionless shock experiment on PLX. Here, $\rho_{i}$ is the thermal ion gyro-radius, $\beta$ the ratio of plasma thermal-to-magnetic pressure, $M_{A}$ the Alfvén Mach number, $\lambda_{i}$ the ion mean free path, $R_{M}$ the magnetic Reynold's number, $\omega_{c i}$ the ion-cyclotron frequency, $\tau_{\text {exp }}$ the shock-transit time, and $c / \omega_{p i}$ the ion inertial length. All values given are for a quasi-perpendicular shock, and all speeds are in the laboratory frame.

If PLX is upgraded to have many more guns, e.g. 36 or 60 , then the merging and spherical convergence of all the jets could produce $\mathrm{cm}-, \mu \mathrm{s}-$, and Mbar-scale plasmas, enabling unique fundamental physics studies of many HED topics (as described in the United States Department of Energy Report of the Workshop on High Energy Density Laboratory Physics Research Needs, Nov. 15-18, 2009) and the exploration of a standoff MIF driver for fusion energy (Hsu et al. 2012a).

\section{Acknowledgements}

We thank Dr. Y. C. F. Thio and Dr. J. T. Cassibry for many useful conversations and Dr. Glen Wurden and Dr. Thomas Intrator for loaning numerous items of laboratory and diagnostic equipment, especially the multiple-frame CCD camera. This work was supported by the Office of Science (Office of Fusion Energy Sciences) and the LANL Laboratory Directed Research and Development (LDRD) Program under U.S. Department of Energy contract no. DE-AC52-06NA25396.

\section{REFERENCES}

Adams, C. S., Lynn, A. G., Gilmore, M. A., Merritt, E. C., Moser, A. L. and Hsu, S. C. 2012 Schlieren imaging diagnostic for a collisionless shock experiment. Bull. Am. Phys. Soc. 57, 130.

Awe, T. J., Adams, C. S., Davis, J. S., Hanna, D. S., Hsu, S. C. and Cassibry, J. T. 2011 Onedimensional radiation-hydrodynamic scaling studies of imploding spherical plasma liners. Phys. Plasmas 18, 072705.

Baker, D. A. and Hammel, J. E. 1965 Experimental studies of the penetration of a plasma stream into a transverse magnetic field. Phys. Fluids 8, 713.

Batteh, J. H. 1991 Review of armature research. IEEE Trans. Magn. 27, 224. 
Bellan, P. M., You, S. and Hsu, S. C. 2005 Simulating astrophysical jets in laboratory experiments. Astrophys. Space Sci. 298, 203.

Casanova, M., Larroche, O. and Matte, J.-P. 1991 Kinetic simulation of a collisional shock wave in a plasma. Phys. Rev. Lett. 67, 2143.

Cassibry, J. T., Stanic, M. and Hsu, S. C. 2013 Ideal hydrodynamic scaling relations for a stagnated imploding spherical plasma liner formed by an array of merging plasma jets. Phys. Plasmas 20, 032706 .

Davis, J. S., Hsu, S. C., Golovkin, I. E., MacFarlane, J. J. and Cassibry, J. T. 2012 Onedimensional radiation-hydrodynamic simulations of imploding spherical plasma liners with detailed equation-of-state modeling. Phys. Plasmas 19, 102701.

Degnan, J. H. et al. 2013 Recent magneto-inertial fusion experiments on the field reversed configuration heating experiment. Nucl. Fusion 53, 093003.

Drake, R. P. 2000 The design of laboratory experiments to produce collisionless shocks of cosmic relevance. Phys. Plasmas 7, 4690.

Drake, R. P. 2006 High-Energy-Density-Physics. Berlin: Springer.

Fiksel, G., Fox, W., Bhattacharjee, A., Barnak, D. H., Chang, P.-Y., Germaschewski, K., Hu, S. X. and Nilson, P. M. 2014 Magnetic reconnection between colliding magnetized laser-produced plasma plumes. Phys. Rev. Lett. 113, 105003.

Fox, W., Fiksel, G., Bhattacharjee, A., Chang, P.-Y., Germaschewski, K., Hu, S. X. and Nilson, P. M. 2013 Filamentation instability of counterstreaming laser-driven plasmas. Phys. Rev. Lett. 111, 225002.

Gourdain, P.-A. and Seyler, C. E. 2013 Impact of the hall effect on high-energy-density plasma jets. Phys. Rev. Lett. 110, 015002.

Haas, D. M. et al. 2011 Supersonic jet formation and propagation in x-pinches. Astrophys. Space Sci. 336, 33.

Hsu, S. C. 2009 Technical summary of the first U.S. plasma jet workshop. J. Fusion Energy 28, 246.

Hsu, S. C. et al. 2012a Spherically imploding plasma liners as a standoff driver for magnetoinertial fusion. IEEE Trans. Plasma Sci. 40, 1287.

Hsu, S. C. and Bellan, P. M. 2005 On the jets, kinks, and spheromaks formed by a planar magnetized coaxial gun. Phys. Plasmas 12, 032103.

Hsu, S. C. et al. 2012b Experimental characterization of railgun-driven supersonic plasma jets motivated by high energy density physics applications. Phys. Plasmas 19, 123514.

Intrator, T. et al. $2004 \mathrm{~A}$ high density field reversed configuration (FRC) target for magnetized target fusion: first internal profile measurements of a high density FRC. Phys. Plasmas 11, 2580-2585.

Jaffrin, M. Y. and Probstein, R. F. 1964 Structure of a plasma shock wave. Phys. Fluids 7, 1658.

Ji, H., Toyama, H., Yamagishi, K., Shinohara, S., Fujisawa, A. and Miyamoto, K. 1991 Probe measurements in the REPUTE-1 reversed field pinch. Rev. Sci. Instrum. 62, 2326.

Kirkpatrick, R. C., Lindemuth, I. R. and Ward, M. S. 1995 Magnetized target fusion: an overview. Fusion Tech. 27, 201.

Knapp, C. E. and Kirkpatrick, R. C. 2014 Possible energy gain for a plasma-liner-driven magnetoinertial fusion concept. Phys. Plasmas 21, 070701.

Li, C. K. et al. 2013 Structure and dynamics of colliding plasma jets. Phys. Rev. Lett. 111, 235003.

Lindemuth, I. R. and Kirkpatrick, R. C. 1983 Parameter space for magnetized fuel targets in inertial confinement fusion. Nucl. Fusion 23, 263.

Lindemuth, I. R. and Siemon, R. E. 2009 The fundamental parameter space of controlled thermonuclear fusion. Am. J. Phys. 77, 407.

Liu, W. and Hsu, S. C. 2011 Ideal magnetohydrodynamic simulations of unmagnetized dense plasma jet injection into a hot strongly magnetized plasma. Nucl. Fusion 51, 073026.

Lynn, A. G., Merritt, E., Gilmore, M., Hsu, S. C., Witherspoon, F. D. and Cassibry, J. T. 2010 Diagnostics for the plasma liner experiment. Rev. Sci. Instrum. 81, 10E 115.

Merritt, E. C., Lynn, A. G., Gilmore, M. A. and Hsu, S. C. 2012a Multi-chord fiber-coupled interferometer with a long coherence length laser. Rev. Sci. Instrum. 83, 033506.

Merritt, E. C., Lynn, A. G., Gilmore, M. A., Thoma, C., Loverich, J. and Hsu, S. C. 2012b Multi-chord fiber-coupled interferometry of supersonic plasma jets. Rev. Sci. Instrum. 83, $10 \mathrm{D} 523$. 
Merritt, E. C., Moser, A. L., Hsu, S. C., Adams, C. S., Dunn, J. P., Holgado, A. M. and Gilmore, M. 2014 Experimental evidence for collisional shock formation via two obliquely merging supersonic plasma jets. Phys. Plasmas 21, 055703.

Merritt, E. C., Moser, A. L., Hsu, S. C., Loverich, J. and Gilmore, M. 2013 Experimental characterization of the stagnation layer between two obliquely merging supersonic plasma jets. Phys. Rev. Lett. 111, 085003.

Moser, A. L. and Bellan, P. M. 2012 Magnetic reconnection from a multiscale instability cascade. Nature 482, 379.

Moser, A. L. and Hsu, S. C. 2014 Observation of ionization-mediated transition from collisionless interpenetration to collisional stagnation during merging of two supersonic plasmas. submitted; http://arxiv.org/abs/1405.2286.

Perkins, L. J., Ho, S. K. and Hammer, J. H. 1988 Deep penetration fuelling of reactor-grade tokamak plasmas with accelerated compact toroids. Nucl. Fusion 28, 1365.

Romero-Talamás, C. A., Bellan, P. M. and Hsu, S. C. 2004 Multielement magnetic probe using commercial chip inductors. Rev. Sci. Instrum. 75, 2664.

Ross, J. S., Park, H.-S., Berger, R., Divol, L., Kugland, N. L., Rozmus, W., Ryutov, D. and Glenzer, S. H. 2013 Collisionless coupling of ion and electron temperatures in counterstreaming plasma flows. Phys. Rev. Lett. 110, 145005.

Sagdeev, R. Z. and Kennel, C. F. 1991 Collisionless shock waves. Sci. Am. 264, 106.

Santarius, J. F. 2012 Compression of a spherically symmetric deuterium-tritium plasma liner onto a magnetized deuterium-tritium target. Phys. Plasmas 19, 072705.

Settles, G. S. 2001 Schlieren and Shadowgraph Techniques. New York: Springer.

Slutz, S. A., Herrmann, M. C., Vesey, R. A., Sefkow, A. B., Sinars, D. B., Rovang, D. C., Peterson, K. J. and Cuneo, M. E. 2010 Pulsed-power-driven cylindrical liner implosions of laser preheated fuel magnetized with an axial field. Phys. Plasmas 17, 056303.

Swadling, G. F. et al. 2014 Interpenetration, deflection, and stagnation of cylindrically convergent magnetized supersonic tungsten plasma flows. Phys. Rev. Lett. 113, 035003.

Thio, Y. C. F., Knapp, C. E., Kirkpatrick, R. C., Siemon, R. E. and Turchi, P. J. 2001 A physics exploratory experiment on plasma liner formation. J. Fusion Energy 20, 1.

Thio, Y. C. F., Panarella, E., Kirkpatrick, R. C., Knapp, C. E., Wysocki, F., Parks, P. and Schmidt, G. 1999 Magnetized target fusion in a spheroidal geometry with standoff drivers. In: Proc. of the Second Int. Symp. on Current Trends in Int. Fusion Research, (ed. E. Panarella). Ottawa: National Research Council of Canada, p. 113.

Thoma, C., Welch, D. R. and Hsu, S. C. 2013 Particle-in-cell simulations of collisionless shock formation via head-on merging of two laboratory supersonic plasma jets. Phys. Plasmas 20, 082128.

Witherspoon, F. D., Case, A., Messer, S. J., Bomgardner, II, R., Phillips, M. W., Brockington, S. and Elton, R. 2009 A contoured gap coaxial plasma gun with injected plasma armature. Rev. Sci. Instrum. 80, 083506. 